\title{
In situ Studies of the Reaction-Driven Restructuring of Ni-Co Core-Shell Nanoparticles
}

\author{
Cecile S. Bonifacio ${ }^{1}$, Huolin L. Xin ${ }^{2}$, Sophie Carenco ${ }^{3}$, Miquel Salmeron ${ }^{3}$, Eric A. Stach ${ }^{2}$, and Judith C. \\ Yang $^{1}$ \\ 1. Department of Chemical and Petroleum Engineering, University of Pittsburgh, Pittsburgh, PA 15260 \\ 2. Center for Functional Nanomaterials, Brookhaven National Laboratory, Upton, NY 11973 \\ 3. Materials Science Division, Lawrence Berkeley National Laboratory, Berkeley, CA 94720
}

Core-shell bimetallic nanoparticles (NPs) have novel catalytic, optical and electronic properties compared to their monometallic equivalents [1]. These catalytic properties can be controlled by finetuning chemical composition, surface oxidation, structure and dimension [2,3]. In fact, segregation in the core-shell structure has been demonstrated as a potential route of tuning the NPs catalytic properties through in situ gas reaction studies [4]. However, experimental evidence of elemental distributions during reactions is necessary to confirm this hypothesis. Here we have used in situ imaging and spectroscopy techniques provide direct evidence of the structural and elemental changes which occur in these NPs during reactions.

Oxidation and reduction at 0.3 Torr in $\mathrm{O}_{2}$ and $\mathrm{H}_{2}$ gas at $220^{\circ} \mathrm{C}$ and $270^{\circ} \mathrm{C}$, respectively, of the core-shell $\mathrm{Ni}$-Co nanoparticles (NPs) were performed in two cycles in using both an environmental transmission electron microscope (ETEM) and an ambient pressure x-ray photoelectron spectrometer (AP-XPS). These NPs (diameter of $25 \mathrm{~nm}$ ) were dispersed in hexane and drop-casted on SiN and Si grids for the in situ studies. An aberration-corrected FEI Titan ETEM microscope with a Gatan parallel electron energy loss spectrometer (EELS) operated at $300 \mathrm{KV}$ was used for simultaneous imaging, selected area electron diffraction (SAED) pattern and EELS acquisition. SAED patterns and EELS maps provided the structural and elemental distributions of $\mathrm{Ni}$ and $\mathrm{Co}$ within the particle for quantitative analysis.

Figure 1 shows the change in the surface structure of core-shell nanoparticles during the two cycles of oxidation (I-O2 and III-O2) and reduction (II-H2 and IV-H2) in the ETEM [5]. Small clusters (marked with arrows in Figure 1) formed after the $1^{\text {st }}$ reduction cycle and remained on the surface until the end of the experiment. These clusters were identified as cobalt oxide based on the acquired EELS maps (Figure 2a-b). Such results correlate with the increase in Co peak area observed during the $1^{\text {st }}$ cycle of reduction using the surface-sensitive technique of XPS[6] (Figure 2c). Further oxidation and reduction of the particles, labeled III-O2 and IV-H2, respectively, led to detection of Ni species at the surface of the particles (via EELS, Figure 3a and b) and which is correlated to an increase in Ni XPS peak area (Figure 3c). These results clearly show a reaction-driven restructuring of the core-shell NPs by Ni segregation during the $2^{\text {nd }}$ reduction cycle.

Quantitative analysis of the EELS results is underway to identify the valence states during the oxidationreduction reactions. Correlation of the core-shell reconstruction with the electronic structure changes from ETEM and AP-XPS will be provided to obtain the optimum reaction conditions, i.e., catalytic properties, of the Ni-Co core-shell NPs in challenging reactions such as selective $\mathrm{CO}_{2}$ reduction [7].

\section{References:}

[1] S. Alayoglu et al, Nature Materials 7 (2008), p. 333

[2] R. Tiruvalam et al, Faraday Discussions 152 (2011), p. 63

[3] S. Mandal, KM Krishnan, Journal of Materials Chemistry 17 (2007), p. 372 
[4] F. Tao et al, Science 322 (2008) p. 932-934

[5] CS Bonifacio et al, Manuscript in preparation

[6] S. Carenco et al, Small (2015), In press.

[7] CB and JY acknowledge financial support by DOE Basic Energy Sciences (DOE-BES) and the Center for Functional Nanomaterials (CFN) at Brookhaven National Lab supported by the Office of Basic Energy Sciences of the US Department of Energy Contract No. DE-SC0012704. SC was supported by the Director, Office of Science, Office of Basic Energy Sciences, Chemical Sciences, Geosciences, and Biosciences Division, under the Department of Energy Contract No. DE-AC02$05 \mathrm{CH} 11231$.
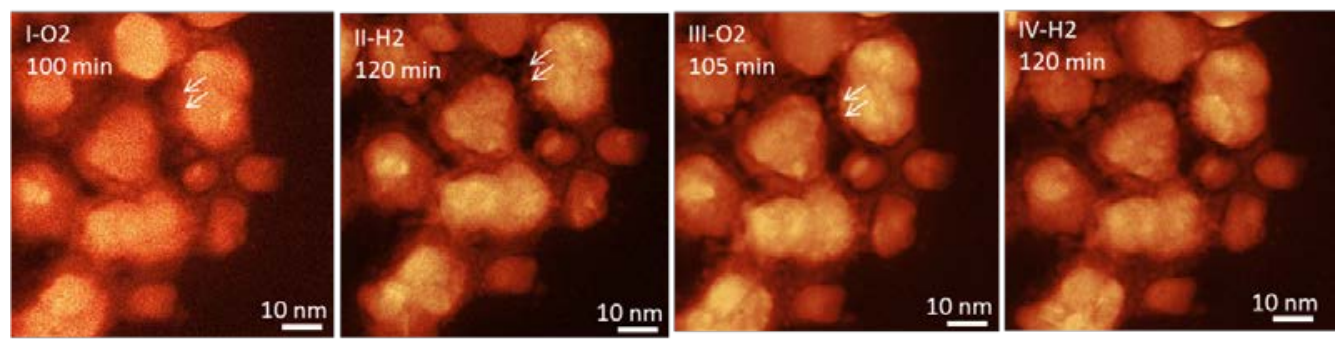

Figure 1. High angle annular dark field (HAADF) images of the core-shell NPs during the oxidation (I$\mathrm{O} 2$ and III-O2) and reduction (II-H2 andIV-H2) cycles. Formation of small clusters (labelled with arrows) on the surface was observed after II-H2 reduction cycle.
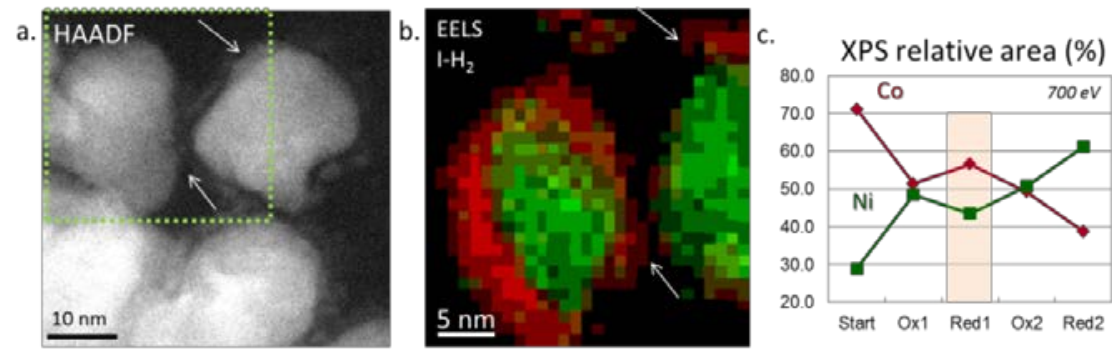

Figure 2. HAADF (a) shows small clusters outside the shell during I-H2 which were identified as Co based on the analysis of the EELS map (b). Clusters of red pixels on the NP surface in (b) verified the Co cluster formation. The increase in Co signal in the XPS $1^{\text {st }}$ reduction cycle (Red1) (c) correlated with the HAADF and EELS results.
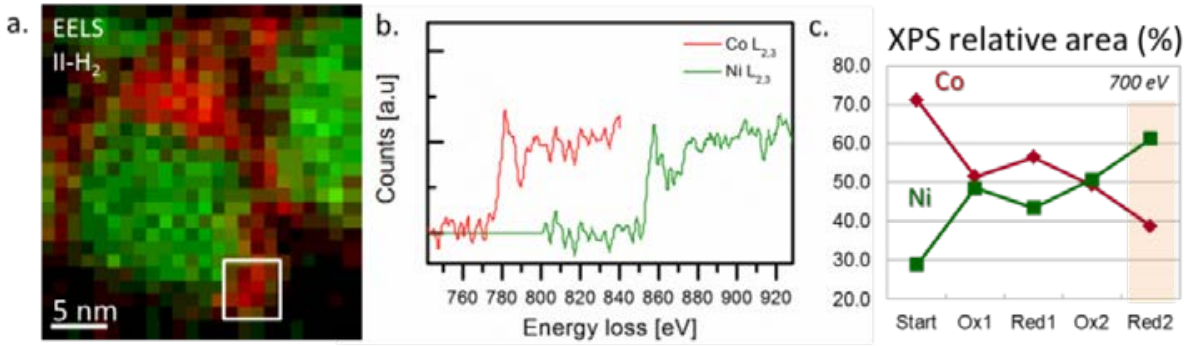

Figure 3. EELS map (a) during II-H2 showing green pixels, Ni species, on the surface of the NP. EELS Co and Ni L2,3 edges (b) were extracted from the marked area in (a) showing significant Ni signals. Such results correlate to the increase in XPS relative area from $\mathrm{Ni}$ (c) indicating Ni segregation to the surface. 